\title{
VARICOSE VEINS
}

\author{
John T Hobbs
}

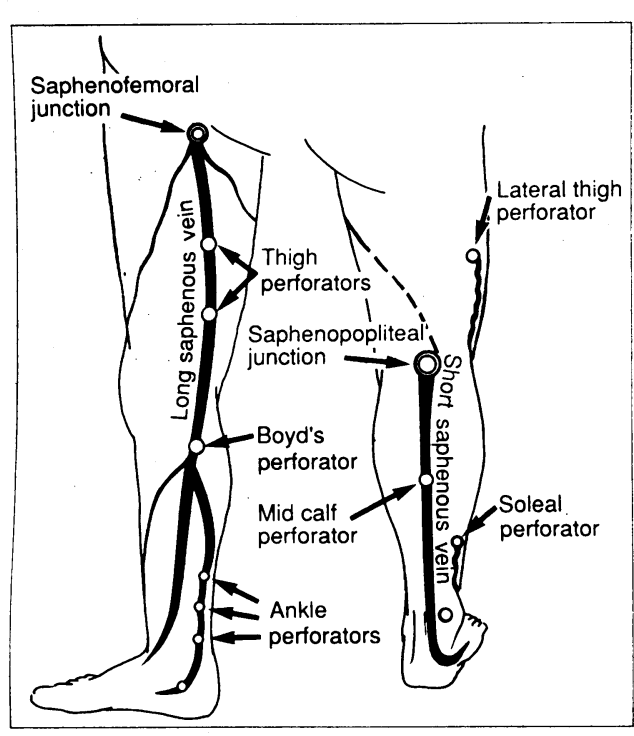

Clinically important veins of the leg: medial view (left) and posterolateral view (right).

\section{Presentation and aetiology}

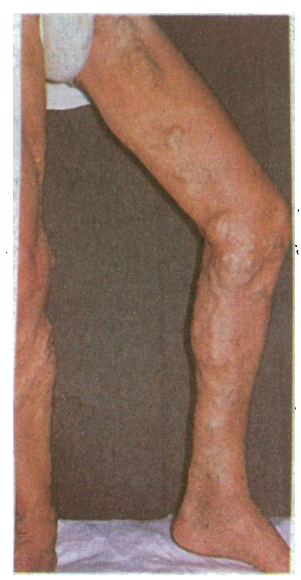

Primary varicose veins affecting the long saphenous system.

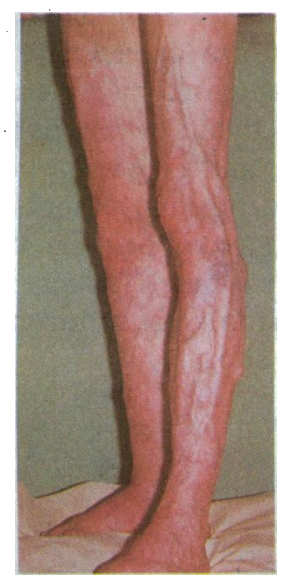

Over 50000 patients are admitted to British hospitals for treatment of varicose veins or their complications each year. Varicose veins cause much morbidity and their incidence is increasing, probably because of the modern Western diet and sedentary lifestyle. "Varicose" veins are dilated, lengthened, and tortuous superficial veins. Although they are sometimes thought of as "vanity" veins, the term "chronic venous insufficiency" gives a more accurate sense of the clinical problem.

In the United Kingdom two thirds of the adult population may show a venous problem at some time, and each year roughly half a million people (the female to male ratio is $5: 1$ ) consult their general practitioners about varicose veins. Because venous disease is seldom acute or life threatening these disorders have a low priority and are usually seen and treated by the least experienced member of the surgical team, often without supervision.

In addition to relief of symptoms and prevention of complications, the cosmetic aspects are important; women seek advice because of disfigurement and also because of complications they see in older people. Treatment is often inadequate or incorrect. If venous problems are carefully assessed and treated the symptoms can be relieved, in most cases with good long term results.

\section{Types of vein}

Athletic veins-Normal veins that are prominent on healthy muscular legs in non-obese people.

Dilated venules (also known as thread veins and spider bursts). These are the result of hormonal effects on soft skin and appear at the menarche, during pregnancy, with the menopause, and at other times of hormonal disturbance.

Primary varicose veins - These are often familial and caused by valvulvar incompetence arid weakness of the vein wall.

Secondary varicose veins - These usually follow deep vein thrombosis as the post-thrombotic syndrome. More rarely they are a manifestation of arteriovenous shunting, usually the Klippel-Trenaunay syndrome but occasionally occurring as a result of trauma by gunshot, stab, or surgery.

\section{Anatomy}

The blood supply to the legs is considerable to meet the demands of active muscles and large bones, and this blood must be returned to the heart through the veins against gravity. The venous system of the legs comprises a superficial system in the skin and subcutaneous fat, and a deep system beneath the fascia. The superficial system is a venous network with prominent long and short saphenous veins, and these and other perforating veins pass through the deep fascia to join the deep veins. The superficial veins, the perforating veins, and the deep veins contain valves to prevent backflow. 


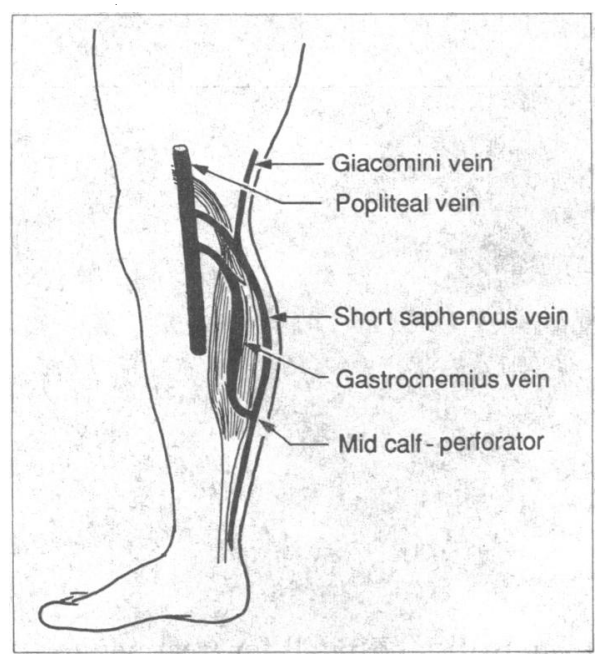

Anatomy of the short saphenous system.

Signs, symptoms, and complications of chronic venous insufficiency

- Unsightly varicose veins

- Tired, aching, heavy legs

- Ankle swelling

- Night cramps

- Restless legs

- Superficial thrombophlebitis

- Haemorrhage

- Irritation and venous eczema

- Lipodermatosclerosis

- Venous ulceration

\section{Investigations}
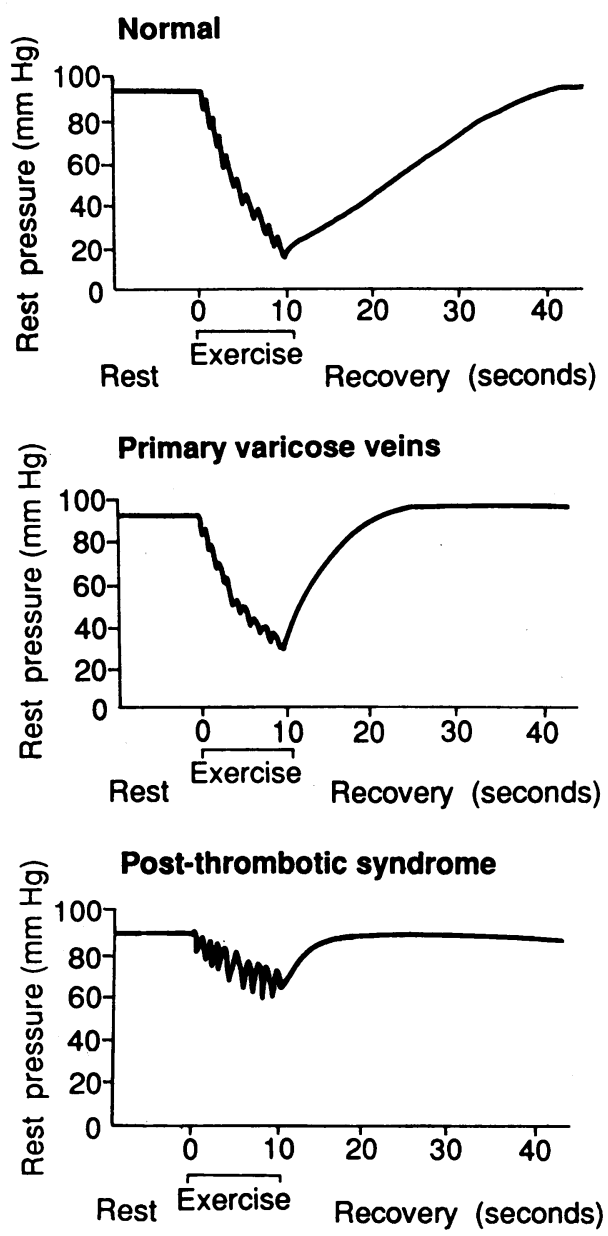

\section{Physiology}

Blood is returned from the periphery by the pumping action of the muscles, which compress the deep veins that contain one way valves, and this is aided by the respiratory movements of the diaphragm. The chief muscular pumps are the muscles of the calf and foot. When deep veins are emptied, reflux is prevented by the valves and blood is sucked in from the superficial veins. When standing the pressure at the ankle is about $90 \mathrm{~mm} \mathrm{Hg}$, the height of a column of blood up to the heart. Muscular contractions of the calf reduce this pressure stepwise to a low plateauknown as the ambulatory venous pressure. At rest after exercise the pressure rises to the level before exercise.

In patients with primary varicose veins the drop in pressure is the same as in a normal leg, but the refilling time is much shorter. In patients with secondary varicose veins the pressure drop is much less than normal and is related to the severity of the venous damage.

Dilatation of superficial veins results in cosmetic disfigurement and occasional irritation. The most common symptoms are tiredness with aching, throbbing, "heavy" legs. They are worse when standing, in hot weather, before menstruation, and are relieved by walking. As soon as muscle activity ceases, however, the pressure rapidly rises again and the symptoms return.

Incompetence of the venous valves permits reflux and so the veins dilate and the pressure patterns become abnormal. The veins then become tortuous and varicose. Pregnant women are particularly prone to developing varicose veins because of the changes in blood hormone concentrations cause the vein walls to relax and dilate during the early stages of pregnancy, and later this dilatation is aggravated by the high pelvic blood flow. Additional factors are the retention of fluid and increased blood volume. Finally, there is considerable compression due to the enlarged uterus.

Many vascular laboratories have been established, and after initially being interested in arterial disease, they are now routinely investigating the venous system.

Measuring venous pressure is the most reliable way of studying venous haemodynamics but it is invasive. Many non-invasive methods have been introduced, including thermography, isotope phlebography, impedance plethysmography, air plethysmography, foot volumetry, photoplethysmography, light reflection rheography, Doppler ultrasonography, B mode ultrasonography, and, most recently, colour flow imaging. At present the best guide is a careful history and physical examination (including the use of a simple Doppler probe) combined with special investigations if these are inconclusive. The sites of incompetent perforating veins are best visualised by peroperative varicography.

The graphs show measurements of venous pressure in a normal leg, a leg with primary varicose veins, and a leg with the post-thrombotic syndrome. The exercise consisted of 10 tiptoe movements. The curves illustrate the fall in pressure in the normal leg and in primary varicose veins, but in the normal leg the recovery time is much longer. Because of the deep valvular incompetence in the post-thrombotic syndrome the drop in pressure is small and the recovery time short. 


\section{Treatment}

\section{Management of varicose veins}

- Reassurance-including camouflage

- Support-elastic stockings

- Sclerotherapy

- Operation

\section{Aide memoire for prescribing stockings}

Q-Quantity: number of items

A-Article: type of stocking (drug tariff only "below knee" or "thigh" length; European Commission allows much greater range)

C-Compression: compression class I-

14-17 $\mathrm{mm} \mathrm{Hg}$; class II-18-24 $\mathrm{mm} \mathrm{Hg}$; class III-25-35 mm Hg; class IV $->35 \mathrm{~mm} \mathrm{Hg}$ is available only from hospitals

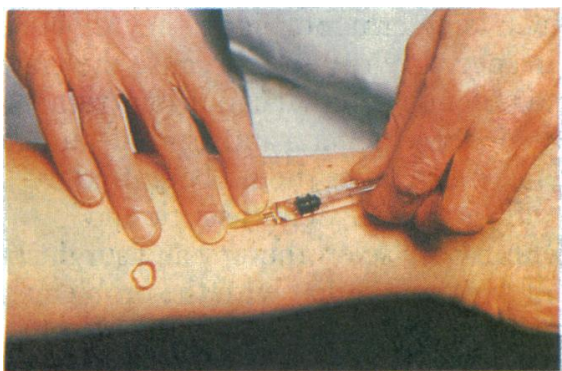

Marking of calf varicosities and injection of sclerosant.

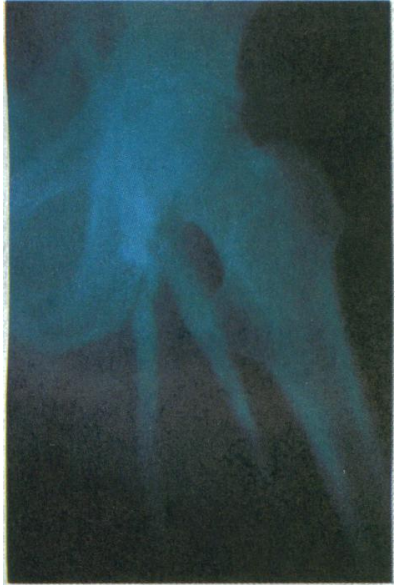

Varicogram showing recurrence at saphenofemoral junction (the needle marks previous incision).

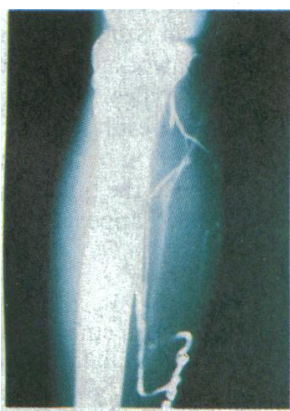

Varicogram showing saphenopopliteal junction with varix.
Not all leg symptoms are due to varicose veins, and some patients referred because of varicose veins do not require treatment for these as the real problem is an orthopaedic or arterial disorder.

During pregnancy, treatment should be conservative with appropriate but cosmetically acceptable support tights. If there is gross incompetence of the saphenofemoral junction causing much pain in the leg or vulval area, or an associated superficial thrombophlebitis, the saphenofemoral junction can be safely and simply divided under local anaesthesia, with immediate relief of symptoms.

\section{Hosiery}

Elastic stockings relieve symptoms, conceal veins, and prevent deterioration, but are not curative. Strong compression on the lower leg is required to prevent complications and should be graduated from the ankle, where problems are most common.

Types available range from those designed mainly for comfort and support to high strength $(40 \mathrm{~mm} \mathrm{Hg})$ medical stockings for control of the disabling post-thrombotic syndrome, and for compression after treatment by injection.

Methods of measuring the pressure exerted by stockings are now available and a British Standards Institution Committee (BS 6612.85) has recommended that the pressure must be graduated. The recommended gradients are that the calf pressure should not be more than $75 \%$ of the pressure exerted at the ankle and the thigh pressure not more than $50 \%$. The publication of this standard prompted the Department of Health to review the "elastic hosiery" section of the Drug Tariff. The aim of the new tariff is to draw attention to the performance of the stockings, and to clarify and simplify prescription.

\section{Sclerotherapy}

Veins can be eliminated either by sclerotherapy or operation and the two methods are complementary. Now that safe sclerosants are available all veins could be treated by injection if compression bandages could be applied and maintained for sufficient time. The aim of injection treatment is to place a small volume of an effective sclerosant in the lumen of the vein, which is then compressed to prevent formation of thrombus (clot). The compression must be maintained until permanent fibrosis has obliterated the lumen. After the initial period when the leg is bandaged, elastic stockings are worn daily until all tenderness, lumps, and discolouration have disappeared.

For large veins a $3 \%$ solution of sodium tetradecyl sulphate is most effective but may cause damage if used for small veins. Laser treatment of dilated venules has been abandoned because of poor results. Venules can be effectively and safely treated by injection, which is best done in a specialist clinic. Although complications are rare, the doctor must always be vigilant as there is a small risk of anaphylaxis. Resuscitation equipment must therefore always be immediately available.

\section{Surgery}

If there is proximal incompetence either in the groin or at the back of the knee operation is the only effective treatment because sclerotherapy rarely gives long lasting successful results at these sites.

Although general (or occasionally epidural) anaesthesia is used, patients are admitted on the day of operation and discharged home the following morning. Simple problems may be dealt with as day cases. The day after operation the bandages are removed and the small incisions covered with strips of permeable non-woven surgical synthetic adhesive tape. Medium strength elastic stockings are then worn during the day for a week until the stitches are removed. The stockings are removed at night for bathing and are left off overnight. Any residual veins can be dealt with by sclerotherapy. 


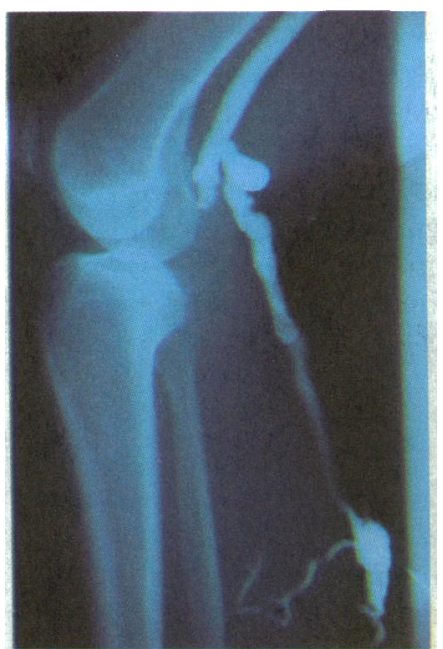

Varicogram showing perforating vein of the lower leg.

\section{Complications}

Superficial thrombophlebitis is often confused with the potentially more dangerous deep vein thrombosis and treated with bed rest and potent anticoagulants. A firm bandage and instructions to walk are all that are required. Because of the signs of inflammation antibiotics are often prescribed, though bacterial infection never occurs in superficial thrombophlebitis. If there is an intravascular clot it should be evacuated by stabbing with a No 11 scalpel blade after applying a blob of local anaesthetic. Rarely the phlebitis spreads proximally to the groin and if seen within the first week may be dealt with by simple operation. This requires only an overnight admission, gives immediate relief, and is a means of long term cure; the pain of operation is less in both extent and duration than the pain that occurs when the condition is treated conservatively.

Venous ulcers are often treated as they were during the previous century but with the additional use of expensive local dressings which are not necessary and may even aggravate the condition. Patients are sometimes seen and treated by district nurses, often without supervision from the general practitioner or hospital consultant. Venous ulcers are fully discussed in the article on leg ulcers.

\section{Conclusions}

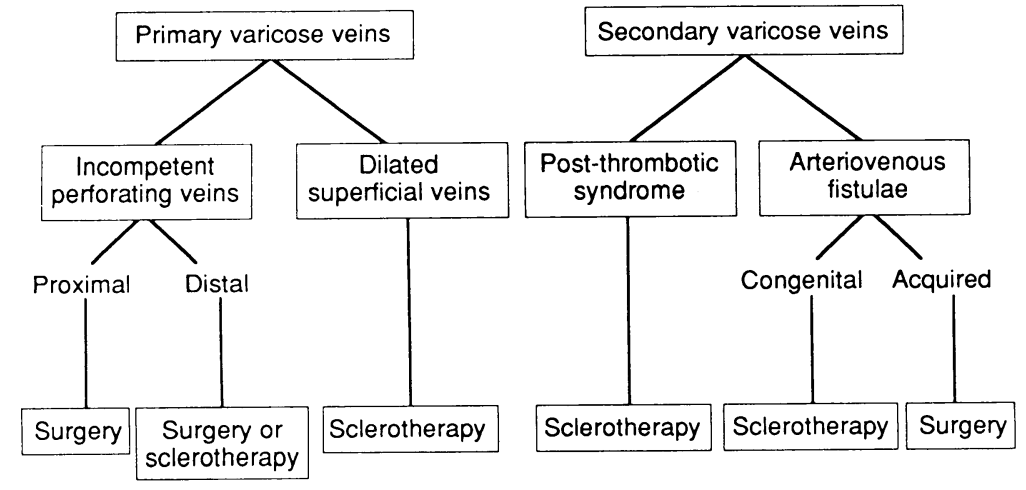

Management of varicose veins.
Because venous problems are so common and cause much long term morbidity they should be treated in special vein clinics because the more acute and potentially lethal conditions rightly take precedence in general and arterial surgical clinics. The vein clinics should be staffed by doctors who are equally enthusiastic and competent in both sclerotherapy and surgical treatment and are familiar with the problems. Consistently good results can be obtained if treatment is precisely planned and properly executed.

We acknowledge with thanks the assistance of the audiovisual department, St Mary's Hospital, London, in the preparation of the illustrations.

Mr John T Hobbs, FRCS, is honorary senior lecturer and formerly was consultant surgeon at St Mary's Hospital, London.

The ABC of Vascular Diseases has been edited by Mr John H N Wolfe, FRCS, consultant vascular surgeon at St Mary's Hospital, London.
During its time in the Strand the fournal had always employed its own compositors. Until 1923 they set by hand the type which was then sent out for printing, machining, and distribution by outside contractors. As we have seen, Odhams had undertaken these tasks since 1911. In fact, their contract was renewed for most of the interwar period, being terminated only in 1937. In 1923 the BMA Council, recognising that the fournal's type had become so dilapidated that it needed urgent replacement, authorised the purchase, at a cost of $£ 4750$, of four Linotype triplex machines "of the most modern pattern" in order to allow machine setting. Hugh Clegg later wrote that "Linotype machines were introduced into this country in 1889 , but it took the $B M \mathcal{F}$ longer probably [sic], than any other periodical to realise that mechanical setting by Linotype was more efficient and more economical." The change greatly affected the compositors "who had been good servants of the Association for many years." But it was achieved without falling foul of their trade union. Indeed, as the chairman of the Journal Committee ironically reported, "The matter has been managed so well that the JOURNAL had had what was probably unique-a compliment paid to it by the general secretary of the Compositors' Union in London for the consideration shown to senior members of the printing staff."

In 1925 the BMA moved from the Strand, where it had been located for almost half a century, to its new home in Tavistock Square. The last issue of the fournal to be put together on the old premises was that of 30 May 1925. During the Whitsun holidays the editorial and printing departments, under Horner's supervision, moved to the new site. The four type setting machines were dismantled and repositioned on the fourth floor of the north east wing, where they were shortly joined by a fifth, capable of setting display advertisements. The new arrangement was less than a year old when the unthinkable occurred - the fournal lost an issue. The reason, of course, was the 1926 General Strike. Odhams' print workers walked out on 4 May and did not return till 17 May. The fournal's typesetters were called out on 6 May and remained on strike for a week. As a result the 8 May issue failed to appear; the next number came out late bearing the double date "May 8th and 15th 1926." This appears to have been the only time in the fournal's history when it failed to make its scheduled appearance in some form or other.

From Mirror of Medicine: A History of the BMF by P W J Bartrip. Published jointly by the $B M F$ and Oxford University Press; BMA members' price UK $£ 29$, overseas $£ 33$, including postage. Obtainable from the Publishing Manager, $B M 7$, PO Box 295, London WC1H 9TE. Non-members UK £35. Obtainable from OUP Distribution Services, Saxon Way West, Corby, Northamptonshire NN18 9ES. 